\title{
Correction to: Novel xylanase producing Bacillus strain X2: molecular phylogenetic analysis and its application for production of xylooligosaccharides
}

\author{
Chandrabhan Dhruw ${ }^{1} \cdot$ Khadim Husain $^{1} \cdot$ Vyas Kumar $^{1} \cdot$ Vijay Chintaman Sonawane $^{1}$ (1)
}

Published online: 9 September 2020

(c) King Abdulaziz City for Science and Technology 2020

\section{Correction to: 3 Biotech (2020) 10:328 \\ https://doi.org/10.1007/s13205-020-02322-1}

Unfortunately, the funding information of the article has been missed in the publication of the article.

The funding information details are provided below:

This work was carried out with the financial support from the Council of Scientific \& Industrial Research, Ministry of science and Technology, India, under the ERIPP project (CSC0131). CD acknowledges Department of Biotechnology, Ministry of science and Technology, India for providing research fellowship for five years. We acknowledge Microbial Type Culture Collection and Gene Bank (MTCC), Chandigarh, for providing the MALDI and GCMS facilities. We thank Dr. Alejandro P Rooney (Supervisory Research Geneticist) and Dr. Christopher A Dunlap (Research Chemist) from USDA ARS NCAUR for very generous help on emphasizing the Bacillus taxonomy.

The original article can be found online at https://doi.org/10.1007/ s13205-020-02322-1.

Vijay Chintaman Sonawane

vijay@imtech.res.in

1 BERPDC, CSIR-Institute of Microbial Technology, Sector 39A, Chandigarh 160036, India 